\title{
超轻元素铍的电子探针定量分析最佳条件探索: 以绿柱石为例
}

张文兰 ${ }^{*}$, 车旭东 ${ }^{1}$, 王汝成 ${ }^{1}$, 谢䂞 ${ }^{1}$, 李晓峰 ${ }^{2,3,4}$, 张迪 $^{5}$

1. 南京大学地球科学与工程学院, 南京大学内生金属矿床成矿机制研究国家重点实验室, 南京 210023;

2. 中国科学院地质与地球物理研究所, 中国科学院矿产资源研究重点实验室, 北京 100029;

3. 中国科学院地球科学研究院, 北京 100029 ;

4. 中国科学院大学地球与行星科学学院, 北京 100049 ;

5. 中国科学院地质与地球物理研究所, 岩石圈演化国家重点实验室, 北京 100029

*联系人, E-mail: zhangwl@nju.edu.cn

2020-03-24 收稿, 2020-05-29 修回, 2020-06-01 接受, 2020-06-02 网络版发表

中国科学院地质与地球物理研究所重点自主部署项目(IGGCAS-201902)、国家自然科学基金(91855209, 41572058)和第二次青藏高原综合科 学考察研究(2019QZKK0802)资助

摘要 金属铍具有耐高温、耐腐蚀特点, 是国防军工和航空航天等领域不可替代的金属材料. 然而, 由于缺乏铍元 素微区无损定量分析技术，制约了诸如铍元素在矿物中赋存状态、铍超常富集机制等相关基础研究和找矿勘查技 术的发展. 虽然电子探针具有矿物微区定量分析的优势, 但是目前还没有有效的对超轻元素Be的分析技术. 本研究 选择日本电子两种不同型号的电子探针(JEOL JXA-8800和JXA-8100), 配置不同分光晶体(LDEB和LDE3H), 对绿 柱石中铍的定量分析方法进行了研究, 结果以加速电压 $10 \mathrm{kV}$ 、束流 $20 \mathrm{nA}$ 、分光晶体为 $\mathrm{LDE} 3 \mathrm{H} 、 \mathrm{Base}=1$, window $=5$ 、slit为 open、微分模式(differential)、峰位测量时间为 $50 \mathrm{~s}$ 、背景测量 $25 \mathrm{~s}$ 、标样为金属Be为最佳条件. 该 方法测试绿柱石中 $\mathrm{Be}$ 的检测限低于 $250 \mathrm{ppm}(1 \mathrm{ppm}=1 \mu \mathrm{g} / \mathrm{g}), \mathrm{BeO}$ 的标准误差 $\sigma=0.52 \mathrm{wt} \%$. 此外, 对造成铍测试困难 的原因、分析过程中出现的现象、条件设置等, 在理论上进行了剖析. 这不仅为同行电子探针测试Be元素提供了 较成熟的测试经验, 而且也为铍资源的相关研究和找矿勘查提供了技术支撑.

关键词电子探针, 定量分析, 超轻元素铍, 绿柱石, 分析条件, 吸收效应

铍属于亲石元素 ${ }^{[1]}$, 在上地壳中克拉克值为 $3 \times 10^{-6}$, 在地壳元素丰度列47位 ${ }^{[2]}$. 铍金属不仅轻, 熔点非常高 $\left(1278^{\circ} \mathrm{C}\right)$, 是所有金属中属热容量最大的一种金属 ${ }^{[3]}$, 而且强度大, 耐腐蚀. 它通常用于国防军工, 如导弹、 火箭和卫星的部件——陀螺仪等，也是制造核反应堆 中子缓冲器主要成分，被认为是“空间金属”和“核子堆 保护神”. 因此, 铍是重要的战略物资, 属于不可替代的 金属, 具有重要的国防和经济价值 ${ }^{[4]}$. 由于 20 世纪 90 年 代以后我国对铍矿产资源勘查研究工作的重视程度不
足，导致铍金属由原先的属于布局优势矿种，变为“被 卡脖子”的矿种. 截至2014年底我国对铍的需求量为 $150 \mathrm{t}$, 而自产量为 $54 \mathrm{t}$, 预测到 2030 年需求量将达到 250 t. 由于我国的铍矿品位普遍低于 $0.25 \%$, 导致 $98 \%$ 的铍矿山因为开采成本过高而无法利用，因此中国的 铍金属主要靠进口，目前我国铍的对外依存度已经高 达 $85 \%{ }^{[5]}$ ，已经由“卡别人脖子金属”成为“被卡脖子的 金属”. 目前, 在对铍矿床研究方面, 还存着成矿理论的 不完善、在矿物中赋存状态不清楚、分选提炼很困难

引用格式: 张文兰, 车旭东, 王汝成, 等. 超轻元素铍的电子探针定量分析最佳条件探索: 以绿柱石为例. 科学通报, 2020, 65: 3205-3216 Zhang W L, Che X D, Wang R C, et al. Optimum conditions for quantitative analysis of beryllium by electron probe microanalysis: A case study of beryl (in Chinese). Chin Sci Bull, 2020, 65: 3205-3216, doi: 10.1360/TB-2020-0316 
等诸多问题 ${ }^{[6]}$. 因此, 加强对铍资源的研究工作显得尤 为重要.

自然界中已发现的铍矿物有近 60 种，其中常见的 有 20 多种. 以硅酸盐类最多, 分布也较广, 其次为磷酸 盐类, 仅有少数为简单氧化物、嗍酸盐、砷酸盐和锑 酸盐等. 主要含铍矿物有绿柱石 $\left(\mathrm{Be}_{3} \mathrm{Al}_{2}\left[\mathrm{Si}_{6} \mathrm{O}_{18}\right]\right)$ 、金绿 宝石 $\left(\mathrm{BeAl}_{2} \mathrm{O}_{4}\right)$ 、硅钪铍石 $\left(\mathrm{Be}_{3} \mathrm{Sc}_{2}\left[\mathrm{Si}_{6} \mathrm{O}_{18}\right]\right)$ 、硅铍石 $\left(\mathrm{Be}_{2}\left[\mathrm{SiO}_{4}\right]\right)$ 、铍石 $(\mathrm{BeO})$ 等, 其中绿柱石和硅铍石最具 有工业价值. 作为最具有工业价值铍矿物之一的绿柱 石, 前人对其开展了较多的矿物晶体结构和谱学方面 的研究 ${ }^{[7,8]}$, 而对其铍的化学成分多为传统的湿化学方 法分析，但这些测试方法都对颗粒的均一性和大小有 极高的要求. 而且给出的数据往往是绿柱石整个颗粒 的化学成分, 不能显示颗粒内部微区原位元素的变化. 激光剥蚀电感耦合等离子质谱(LA-ICP-MS)分析主要 用于矿物微量Be的测试，另外该方法还受到对样品损 伤大，缺少合适标样，空间分辨率不足等问题的困扰， 目前未见用于主要含铍矿物其主量Be测试的报道. 而 电子探针(EPMA)的分析在微束分析方法中, 具有极高 的空间分辨率 $(\sim 3 \mu \mathrm{m})$, 对样品属于无损分析, 可用于矿 物中主量Be的测试. 但是由于受到分析技术难度大因 素的制约, 目前对于绿柱石在EPMA分析过程中, $\mathrm{BeO}$ 一般不分析, 而是根据矿物晶体化学计算获得 ${ }^{[9 \sim 12]}$. 因 此, 由EPMA分析获得的含铍矿物的数据少之又少, 国 内外相关文献报道也很少.

\section{EPMA测试Be研究现状}

Be 是EPMA所能分析的元素 $\left({ }^{4} \mathrm{Be}-{ }^{92} \mathrm{U}\right)$ 范围内最轻 的元素, 测试难度非常大, 以至于存在大多数EPMA分 析人员“遇Be后退”的㘣态.

尽管Be元素在EPMA测试中极为困难, 但前人还 是做了艰苦的尝试. 2001年, Dyar等人 ${ }^{[13]}$ 报道了在德国 波茨坦地学中心(GFZ)实验室用Cameca-SX-50测得的 硅铍石 (phenakite)和硼铍石(Hambergite)中Be的含量, 并给出 $\mathrm{Be}$ 的校正标样是 $\mathrm{Be}$ 金属. 他们给出了部分分析 条件, 但结果的重复性有待提高. 即便如此该成果的发 表, 在EPMA 对Be矿物测试方面也属于里程碑式成果.

2006年，张文兰等人 ${ }^{[14]}$ 以会议增刊的形式初步报 道了电子探针定量分析雪宝顶绿柱石(样品号：BerylB)中Be的测试结果. 该测试利用日本电子JXA-8800 (JEOL), 分光晶体LDEB, 使用金属Be标样, 并对分析 条件和分析过程中遇到的问题进行了简单的讨论. 赵
同新等人 ${ }^{[15]}$ 利用岛津EPMA-1720仪器, 分光晶体 LSA200, 对新疆绿柱石进行过EPMA测试, 也获得了不 错的结果. 浙江大学吴润秋和饶灿 ${ }^{[6]}$ 发表了他们利用岛 津EPMA-1720H, 分光晶体LSA300, 对含Be矿物EPMA 分析成果, 分析了绿柱石、硅铍石和羟硅铍石 3 种矿物, 都取得了不错的结果, 他们为岛津用户积累了经验.

从目前已经进行过 $\mathrm{Be}$ 的电子探针测试的实验室 看, 每个实验室所使用的仪器, 来自不同的厂家, 所用 晶体和修正方法、设置的测试条件, 尤其是脉冲高度 分析器(PHA)的设置方面都不相同, 这为总结出一套成 熟的测试方法, 增加了难度, 因此目前对于Be的EPMA 测试还处于一个探索阶段, 更缺少一套标准样品用来 标定 $^{[13]}$.

本研究寻找并建立了一套利用电子探针分析 $\mathrm{Be}$ 含 量切实可行的测试方法, 除了在加速电压、束流方面 优化选择外, 还在脉冲高度分析器-增益(PHA gain)设 置、标样的选取、试样Be峰位的确定、上下背景的选 择等方面做了详细的介绍, 进而从理论上探讨了 Be测 试困难的机理, 以及优选一些测试条件后, 对测试结 果的影响. 这套测试方法不仅提高了利用电子探针测 试Be含量的精确度和准确度, 而且为各EPMA实验室 Be的测试提供了参考.

\section{2 铍的化学特性及导致EPMA测试困难的 机理}

在元素周期表中, $\mathrm{Be}$ 的原子序数为 4 , 原子量 9.012182, 是仅次于 $\operatorname{Li}(6.941)$ 最轻的碱土金属元素. Be 有两个电子层, $1 \mathrm{~S}$ 和 $2 \mathrm{~S}$, 每层上有 2 个电子, $\mathrm{Be}$ 的化合价 态为 +2 价, Be的氧化物和卤化物都具有共价键特性 ${ }^{[1]}$. 根据电子探针定量分析工作原理，当高压电子束照射 到样品表面后, 会激发出所含元素的特征X射线, 这种 $\mathrm{X}$ 射线, 不但可以获知元素的种类, 还可获得元素的准 确含量. 从Be元素的化学特性而言, 导致EPMA定量分 析困难的机理可归纳为如下 3 方面:

(1) 由于 $\mathrm{Be}$ 元素原子序数为 4 , 核外电子只有 $\mathrm{K}$ 层, 当高压电子束与之作用时, 被人射电子束击中的可能 只有 $\mathrm{K}$ 线系上的 2 个电子. 首先很难打准这两个电子, 即便是打准了而被激发所产生的特征X射线的强度也 很微弱, 这就直接导致Be的计数率很低. 除此之外, $\mathrm{Be}$ 的质量吸收系数大(也称为质量衰减系数), 原始辐射的 衰减系数很大(计数率衰减的非常快 $)^{[15,16]}$, 因此，与其 他元素相比, Be的特征X射线的强度和峰背比 $(\mathrm{P} / \mathrm{B})$ 要 
低得多. $\mathrm{Be}$ 金属的计数率 $(\mathrm{cps} / \mu \mathrm{A})$ 一般在 $10^{4}$ 范围, 其峰 背比在 100 左右. 而较重的元素的计数率在 $10^{6}$, 其峰背 比在几百至一千多, 这就是导致Be元素难以测准的关 键因素之一.

(2) 在电子探针分析领域, 通常以元素波长 $\lambda=12 \AA$ 为界. 波长 $\lambda<12 \AA$ 的元素为能量较高元素, $\lambda>12 \AA$ 的元 素为能量较低的元素或是超轻元素. 超轻元素一般指 $\mathrm{Be} 、 \mathrm{~B} 、 \mathrm{C} 、 \mathrm{~N} 、 \mathrm{O} 、 \mathrm{~F}$ 六种元素, 它们各自波长与能量 分别为: $\operatorname{Be}(113.43 \AA, 0.109 \mathrm{keV}) 、 \mathrm{~B}(67.6 \AA$, $0.183 \mathrm{keV}) 、 \mathrm{C}(44.7 \AA, 0.277 \mathrm{keV}) 、 \mathrm{~N}(31.6 \AA$, $0.392 \mathrm{keV}) 、 \mathrm{O}(23.62 \AA, 0.525 \mathrm{keV}), \mathrm{F}(18.32 \AA$, $0.677 \mathrm{keV})^{[17]}$. 这些超轻元素 $\mathrm{K}$ 系的特征 $X$ 射线都具波 长 $\lambda>12 \AA$ 、能量低 $E_{0}<1 \mathrm{keV}$ 特点. 而Be元素的波长最 长, 能量最低, 这也是导致Be元素难以测准的又一关键 因素.

根据布拉格衍射原理: $2 d \sin \theta=n \lambda$, 对于 $\lambda$ 值最大的 超轻元素Be而言, 要想满足布拉格公式的条件, 使人射 到晶体中X-射线能够产生分光进行元素鉴定, 就需要 面网间距 $2 d$ 值也很大的分光晶体. 因此, 各个仪器厂家 专门为此研制出了具有大面网间距的分光晶体, Came$\mathrm{ca}$ 厂家生产的Be元素分光晶体为 $\mathrm{PC} 3(2 d=20 \mathrm{~nm})$, 岛津 厂家生产Be元素分光晶体为 $\operatorname{LSA} 300(2 d=30 \mathrm{~nm}) . \mathrm{JEOL}$ 厂商目前有两种分光晶体用作 $\mathrm{Be}$ 元素测试, 分别为 $\operatorname{LDEB}(2 d=14.5 \mathrm{~nm})$ 和LDE3H( $2 d=20 \mathrm{~nm})$, 为超轻元素 测试在技术上提供了保障.

(3) 谱线干扰, 对超轻元素而言, 只有 K线系, 而重 元素则存在着 $\mathrm{L}$ 线系和 $\mathrm{M}$ 线系, 以及它们的高次衍射线 系. 这些线系常常在超轻元素 $\mathrm{K}$ 线系附近出现, 容易对 超轻元素的 $\mathrm{K}$ 线系进行干扰, 如 $\mathrm{Si}$ 的 $\mathrm{L}_{2,3}$ 及 $\mathrm{M} 1$ 线系和 $\mathrm{C}$ 的 $\mathrm{K}_{\mathrm{a} 3}$ 线系对 $\mathrm{Be}$ 的 $\mathrm{Ka}$ 线系进行干扰, 导致测试结果不 理想 ${ }^{[16,17]}$. 这也是导致Be元素难测的又一关键因素.

\section{3 实验样品选择与前处理}

分析测试的绿柱石样品采自四川雪宝顶W-Sn-Be 矿床的石英脉中. 晶体为板状, 无色透明, 以(0001)和 (1121) 晶面发育为特征. 晶体颗粒较大 (大于 $2 \mathrm{~cm} \times$ $4 \mathrm{~cm}$ ), 样品编号为B4, 其主量元素和微量元素分析数 据详见Liu等人 ${ }^{[18]}$, 该数据可作为电子探针分析数据的 参考. 本研究测试所用样品为B4号样品的碎片, 将B4样 品碎片用环氧树脂进行包埋、抛光、镀碳、待分析, 碎片大小为 $3 \mathrm{~mm} \times 8 \mathrm{~mm}$.

需要注意的是, 超轻元素的电子探针测试时, 碳膜
的厚度很重要. 由于 $B e$ 的特征 $X$-射线强度很低, 且其 他元素对Be的吸收效应很强, 如果碳膜太厚, 势必影 响对Be-X射线探测到的强度, 如果碳膜太薄, 在分析 过程中, 容易被高压电子束击穿, 使样品表面电荷聚 集而导致无法测量. Zhang和Yang ${ }^{[19]}$ 计算了试样放置 位置与碳棒溅射源之间角度不同对碳膜厚度的影响. 为了保持试样与标样的碳膜厚度一致, 减少试样与标 样由于碳膜厚度不等而出现的误差, 本研究所用试样 与标样, 使用数字化的LEICA-EM-ACE200镀膜仪, 将 试样放在溅射源的正下方, 设定镀膜厚度为 $15 \mathrm{~nm}$, 完 成镀膜.

\section{4 分析条件的选择与优化}

电子探针在进行元素分析时, 是通过测定元素的 特征X射线的计数, 再与已知标样的计数进行对比计 算, 最终由X射线强度换算成元素的浓度, 来获得元素 的质量百分比. 由于超轻元素所产生的特征 X射线稀 疏、量少, 本身其计数率与峰背比都很低. 因此, 超轻 元素在分析时, 首先考虑是如何提高计数率和峰背比 这两个参数, 由于Be元素波长长 $(\lambda=114 \AA$ 、能量低 $E \leqslant$ $1 \mathrm{keV}$ ), 原始衰减很大, 其峰背比很低. 为提高计数率和 峰背比, 在分析条件设置过程中, 需要重点考虑的就是 加速电压和束流.

\section{1 加速电压与束流的选择(分光晶体: LDE3H)}

首先固定束流为 $100 \mathrm{nA}$, 分别选择 $3,5,10,15 \mathrm{kV}$ 四种不同的加速电压, 利用LDE3H分光晶体对绿柱石 进行了精细的Be元素波谱扫描. 扫描条件为步长 20 $\mu \mathrm{m}$, Dwell为 $500 \mathrm{~ms}$, 扫描起始位置为 $L=160 \mathrm{~mm}$, 结束 位置 $L=185 \mathrm{~mm}$, 该范围涵盖了Be整个峰型. 扫描结果 使用仪器中Smooth处理软件, 进行统一参数的处理后 并投图(图1(a)).

其次, 再从每种条件下获得的Be元素扫描图中计数 最大位置作为峰位, 计数最小的位置作为背景, 由于 $\mathrm{Si}$ 的 $\mathrm{L}_{2,3}$ 和 $\mathrm{M}_{1}$ 峰的干扰, 上背景设置需要绕开干扰峰, 而远 离峰位位置, 因此测试时所取位置都取在下背景一侧, 峰位和背景各自测试 $100 \mathrm{~s}$, 然后将其所得的计数进行比 较, 获得峰背比列入表1. 从图1(a)和表1可见 3 和 $5 \mathrm{kV}$ 的 峰型最好, 峰背比也高. 但是这两种电压不适合测试绿 柱石中的其他元素, 故没有选择这两种电压用来测试.

最后, 为了兼顾其他元素的测试, 固定 $10 \mathrm{kV}$ 加速 电压, 分别选择 $10,20,50,100,200 \mathrm{nA}$ 五种不同的束 

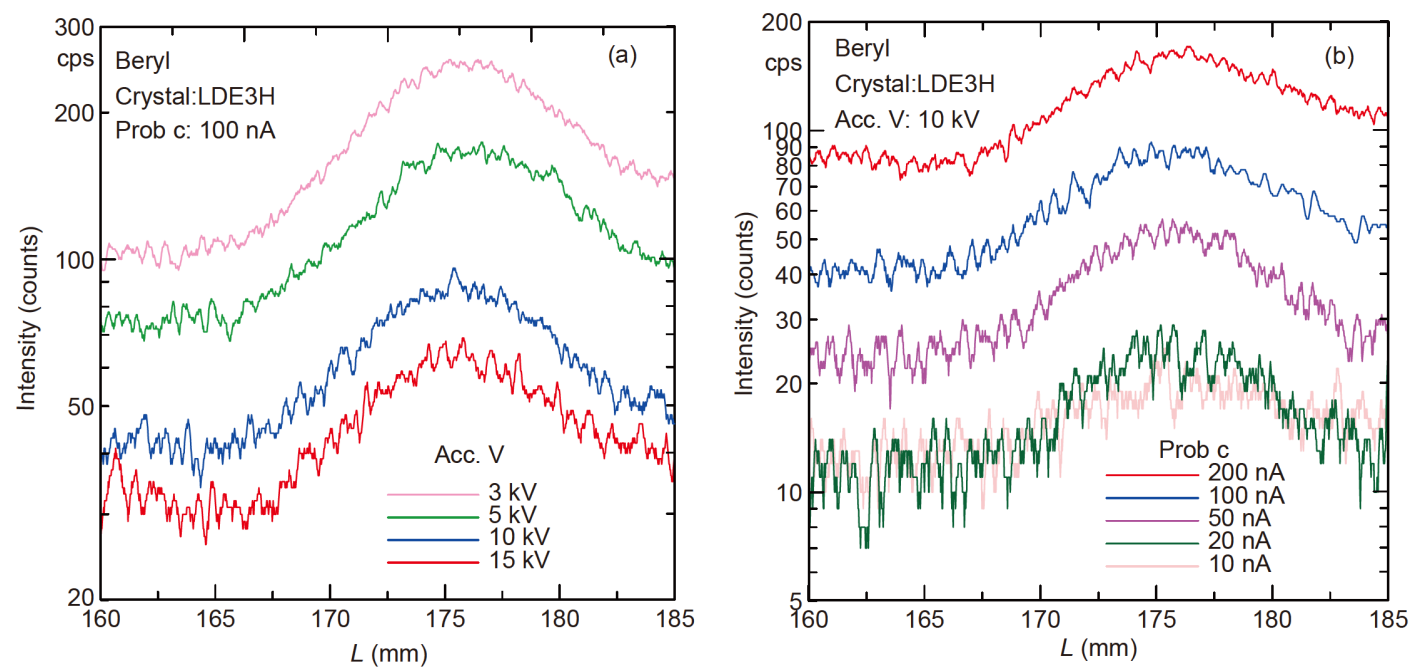

图 1 (网络版彩色)不同条件下绿柱石Be元素的波谱扫描. (a) 束流 $100 \mathrm{nA}$, 不同加速电压条件下绿柱石波谱扫描; (b) 加速电压 $10 \mathrm{kV}$, 不同束流 条件下绿柱石波谱扫描

Figure 1 (Color online) Be element spectral scanning results of beryl under different conditions. (a) Spectral scanning results of beryl under different accelerating voltages on the current of $100 \mathrm{nA}$; (b) spectral scanning results of beryl under different beam currents at the accelerating voltage of $10 \mathrm{kV}$

表 1 不同条件下绿柱石Be元素波谱扫描获得的峰背比值(分光晶体LDE3H)

Table 1 Peak to background ratio of beryl by element spectrum scanning under different conditions (crystal LDE3H)

\begin{tabular}{|c|c|c|c|c|c|c|c|c|c|}
\hline & \multicolumn{4}{|c|}{$100 \mathrm{nA}$ 不同加速电压 $(100 \mathrm{~s})$} & \multicolumn{5}{|c|}{$10 \mathrm{kV}$ 不同束流(100 s) } \\
\hline & $3 \mathrm{kV}$ & $5 \mathrm{kV}$ & $10 \mathrm{kV}$ & $15 \mathrm{kV}$ & $10 \mathrm{nA}$ & $20 \mathrm{nA}$ & $50 \mathrm{nA}$ & $100 \mathrm{nA}$ & $200 \mathrm{nA}$ \\
\hline 峰位计数(cps) & 18317 & 34796 & 15660 & 12485 & 1930 & 3884 & 7883 & 15660 & 36486 \\
\hline 背景计数(cps) & 8431 & 13653 & 7926 & 6795 & 994 & 1766 & 4120 & 7926 & 17684 \\
\hline 峰背比P/B & 2.17 & 2.55 & 1.97 & 1.84 & 1.94 & 2.2 & 1.91 & 1.97 & 2.06 \\
\hline
\end{tabular}

流，对绿柱石进行了精细的Be元素波谱扫描，扫描条 件、数据处理及投图方法同上. 处理后的数据投图(图 1(b)). 由于不同束流扫描后Be最大计数位置和最小计 数位置也不同，因此必须从每种不同束流Be元素扫描 图中, 把计数最大位置作为峰位、计数最小的位置作 为背景(下背景), 各自测试 $100 \mathrm{~s}$, 然后将其所得的计数 进行比较, 获得峰背比列人表 1 .

从表 1 中数据可知 $10 \mathrm{kV} 、 20 \mathrm{nA}$ 获得的峰背比为 2.2 , 在 5 种不同束流条件下其峰背比最高, 这是本研究 实验选择 $10 \mathrm{kV}$ 电压、束流为 $20 \mathrm{nA}$ 依据所在，而且这 个条件还可能满足绿柱石中其他元素的测试. 如果遇 到 $\mathrm{Be}$ 含量更低的铍矿物, 可以考虑使用低电压将 $\mathrm{Be}$ 元 素单独测试.

\section{2 束斑直径的选择(分光晶体LDE3H)}

绿柱石除了 $\mathrm{Be}$ 元素为主量元素外, 还有 $\mathrm{Si} 、 \mathrm{Al}$ 、
$\mathrm{Na} 、 \mathrm{Cs}$ 等主要元素. 对于晶体结构比较疏松、空隙大 的矿物(如: 架状结构和六方柱状结构矿物), $\mathrm{Na}$ 赋存在 这些矿物结构的大空间中, 属于热扩散元素, 在EPMA 测量过程中一般是加大束斑直径，以避免出现计数率 降低现象, 从而导致测量结果不准, 因此在束斑的设置 时也要考虑此因素.

由于Be属超轻元素, 被激发出来的特征X射线具有 少而稀疏的显著特点, 因此束流和束斑直径大小, 对能 否获得足够的特征X射线也是必须考虑的因素. 在对新 疆阿尔泰绿柱石定量分析过程中(ALT- $\left.{ }^{[7]}\right)$, 设置不同 的束流和束斑直径(共8种)测试条件(表2). 结果显示束 斑和束流的变化对 $\mathrm{BeO}$ 测试结果影响不大, 而 $\mathrm{Na}_{2} \mathrm{O}$ 的 含量变化波动却很大. 这可能与 $\mathrm{Be}-\mathrm{O}$ 是以共价键的方 式结合、 $\mathrm{Na}_{2} \mathrm{O}$ 是以离子键方式结合有关(表2). 但考虑 到超轻元素由于吸收效应很大，以及绿柱石中 $\mathrm{Na}$ 元素 的热扩散效应, 结合以往常规元素测量的经验, 本研究 
表 2 阿尔泰3号脉中ALT-1 绿柱石定量测试条件及测量结果 $\left(w t \%\right.$, 分光晶体LDEB, 校正方法ZAF) ${ }^{a)}$

Table 2 Quantitative analytical conditions and results of ALT-1 beryl from Altai No.3 vein (wt\%, crystal: LDEB, correction method ZAF)

\begin{tabular}{|c|c|c|c|c|c|c|c|c|c|c|c|c|c|c|c|c|}
\hline 点号 & 1 & 2 & 3 & 4 & 5 & 6 & 7 & 8 & 9 & 10 & 11 & 12 & 13 & 14 & 15 & 16 \\
\hline 束斑直径( $\mu \mathrm{m})$ & 0 & 0 & 0 & 5 & 10 & 10 & 10 & 5 & 5 & 5 & 5 & 5 & 0 & 0 & 5 & 0 \\
\hline 束流(nA) & 20 & 20 & 20 & 20 & 20 & 20 & 20 & 30 & 30 & 40 & 40 & 40 & 40 & 40 & 50 & 150 \\
\hline 峰位(mm) & 229.1 & 229.1 & 229.1 & 229.1 & 229.1 & 229.1 & 229.1 & 229.1 & 229.1 & 229.1 & 229.1 & 229.1 & 229.1 & 229.1 & 229.1 & 231.7 \\
\hline 上背景(mm) & 0 & 0 & 0 & 0 & 0 & 0 & 0 & 0 & 0 & 0 & 0 & 0 & 0 & 0 & 0 & 0 \\
\hline 下背景(mm) & 30 & 30 & 30 & 30 & 30 & 30 & 30 & 30 & 30 & 30 & 30 & 30 & 30 & 30 & 30 & 30 \\
\hline 峰位计数时间(s) & 100 & 100 & 100 & 100 & 100 & 100 & 100 & 100 & 100 & 100 & 100 & 100 & 100 & 100 & 100 & 100 \\
\hline 背景计数时间(s) & 50 & 50 & 50 & 50 & 50 & 50 & 50 & 50 & 50 & 50 & 50 & 50 & 50 & 50 & 50 & 50 \\
\hline Peak seek W & 0 & 0 & 0 & 0 & 0 & 0 & 0 & 0 & 0 & 0 & 0 & 0 & 0 & 0 & 0 & 1 \\
\hline \multicolumn{17}{|l|}{ 测试结果 $(\mathrm{wt} \%)$} \\
\hline $\mathrm{Na}_{2} \mathrm{O}$ & 2.86 & 1.90 & 1.10 & nd & 1.61 & 4.02 & 2.67 & 1.69 & 1.60 & 1.71 & 1.74 & 3.27 & nd & nd & 1.94 & nd \\
\hline $\mathrm{Al}_{2} \mathrm{O}_{3}$ & 17.59 & 17.53 & 17.64 & nd & 16.84 & 17.05 & 16.82 & 17.60 & 17.41 & 17.66 & 17.40 & 17.40 & nd & nd & 17.65 & nd \\
\hline $\mathrm{SiO}_{2}$ & 64.81 & 66.02 & 64.62 & nd & 65.81 & 65.19 & 65.06 & 65.17 & 65.31 & 64.29 & 64.79 & 65.32 & nd & nd & 65.27 & nd \\
\hline $\mathrm{BeO}$ & 11.21 & 13.79 & 12.11 & 13.85 & 13.17 & 11.85 & 15.86 & 10.74 & 13.14 & 14.07 & 11.17 & 12.82 & 12.88 & 10.57 & 11.97 & 11.44 \\
\hline 共计 & 96.47 & 99.23 & 95.46 & 13.85 & 97.42 & 98.11 & 100.42 & 95.21 & 97.46 & 97.74 & 95.10 & 98.81 & 12.88 & nd & 96.82 & 11.44 \\
\hline D. L. (ppm ) & 9245 & 9099 & 8719 & 7650 & 10070 & 9863 & 7527 & 6292 & 6160 & 4854 & 5763 & 5001 & 5070 & nd & 4623 & 6336 \\
\hline
\end{tabular}

a) ALT-1的湿化学分析数据BeO $=13.35 \mathrm{wt} \%{ }^{[7]}$, 由于LDEB分光晶体Be的峰位 $(L=229.1 \mathrm{~mm})$ 靠近罗兰圆的右端, 故上背景设为 0 , 下背景设 $30 \mathrm{~mm}$; nd: 未测量

选择束斑直径为 $10 \mu \mathrm{m}$ 进行测量.

\section{3 标样的选择与测试}

本研究实验选用的标样为SPI公司的金属Be，原因 之一是由于金属Be标样计数率高、且没有其他元素干 扰, 之二是目前没有合适的含Be矿物标样可选. EPMA定 量分析的规则就是要求标样和试样的测试条件尽量保 持一致, 尤其是加速电压. 为了获取Be元素的准确峰位, 首先用LDE3H分光晶体对金属Be进行3，5，10,15 kV四 种不同的加速电压、束流为 $20 \mathrm{nA}$ 波谱扫描，扫描结果 见图2. 由图可见, 从3, 5, $10 \mathrm{kV}$ 扫描结果看, 随着加速电 压的升高, Be的计数率升高, 而当加速电压升到 $15 \mathrm{kV}$ 时, 计数率反而下降, $10 \mathrm{kV}$ 加速电压计数率最高, 且与绿柱 石波谱扫描条件 $10 \mathrm{kV} 、 20 \mathrm{nA}$ 获得的最好峰背比相吻 合. 因此, 本研究选择了 $10 \mathrm{kV}$ 作为标样的测试电压, Be 金属峰位为 $168.80 \mathrm{~mm}$, 上下背景及测试条件设定见表 3 .

其他元素标样选择如下： $\mathrm{Na}$ 使用美国国家标准局 的钠长石， $\mathrm{Si} 、 \mathrm{Al}$ 和 $\mathrm{Fe}$ 使用美国国家标准局的角闪石， Cs则使用SPI公司的铯榴石标样.

\section{4 绿柱石中Be元素波谱扫描}

由于绿柱石中其他元素的存在，会导致Be的峰位

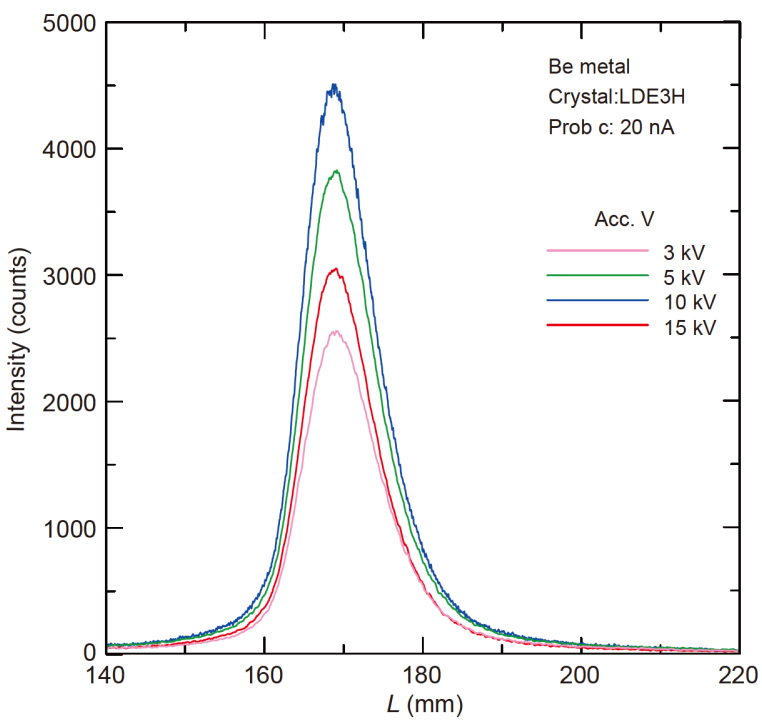

图 2 (网络版彩色)不同加速电压对 $\mathrm{Be}$ 金属的波谱扫描

Figure 2 (Color online) Spectral scanning results of the metal Be at different acceleration voltages with LDE3H crystal

与金属Be的峰位有很大的不同, 因此, 对试样绿柱石进 行Be波谱扫描很有必要，以便确定绿柱石中Be准确峰 位和上下背景．波谱扫描条件为加速电压 $10 \mathrm{kV}, 100$ $n A$ 大束流，以便使Be峰位突出，利于Be峰位确定和上 下背景位置的选择(图3)，其他条件见表3. 从图3可知, 
Be的峰型扁平, 因此在峰位的选择时, 寻找计数最大的 地方, 作为Be的峰位, 由此获得的峰位 $L=174.76 \mathrm{~mm}$, 此 处的计数最高, 为 $92 \mathrm{cps}$. 在背景的选择时, 首先是将 $\mathrm{Be}$ 峰位左侧的背景放大后, 选择计数最低的位置作为下 背景位置 $(L=160.30 \mathrm{~mm}, 38 \mathrm{cps})$. 在上背景的选择时由 于受右侧 $S i$ 的 $L_{2,3}$ 和 $M_{1}$ 峰的干扰, 不能直接在Be峰的右 侧选择, 而是绕开 $\mathrm{Si}$ 的峰在其右侧, 选择没有干扰峰的 地方, 作为上背景位置 $(L=216.56 \mathrm{~mm})$.

\section{5 绿柱石定量分析参数的选定与分析结果}

根据上述分析条件的选择与优化，本次定量分析 条件即要考虑超轻元素Be具有强的吸收效应, 又要考 虑计数率和峰背比, 还要兼顾其他元素能够被激发出 来这些因素, 使用JXA-8100(LDE3H晶体, $2 d=20 \mathrm{~nm}$ ) 定 量分析, 设置的加速电压 $10 \mathrm{kV}$, 束流为 $20 \mathrm{nA}$, Peak Seek设置为 1 , 峰位与背景测量时间分别为 50 和 $25 \mathrm{~s}$, 校 正方法ZAF, 其他参数设置见表 3 , 分析结果列于表 4 .

为了对比, 使用JXA-8800(分光晶体LDEB, $2 d$ $=14.5 \mathrm{~nm}$ ). 对新疆阿尔泰绿柱石(ALT-1)进行了电子探 针定量分析(数据见表2), 其分析条件为固定加速电压 $10 \mathrm{kV}$, 选择不同束流、不同束斑直径 8 种情况. 选用雪 宝顶绿柱石 (Beryl-B)为标样, 该样品数据由比利时大 学矿物研究所Jean-Maris Speetjens 博士用湿化学方法 获得, $\mathrm{BeO}$ 含量为 $11.90 \mathrm{wt}^{\left[{ }^{[7]}\right.}$, 其他测试参数也见表 2 . 从表中数据可知, 束斑和束流变化对测试结果影响不 大, 但是由于同一种条件测试数据偏少, 测量的元素种 类不全, 甚至有的测点只记录了 $\mathrm{BeO}$ 的含量. 但从表 2 与 表 4 中 BeO wt\%数据的稳定性、最低探测极限两项指 标分析, 可以看出 LDE3H分光晶体明显好于LDEB分 光晶体.

\section{6 讨论}

\subsection{Be元素峰位向右漂移现象及产生机理}

相对于 $\mathrm{Be}$ 金属而言, $\mathrm{Be}$ 的氧化物(含 $\mathrm{Be}$ 矿物)在 EPMA测试过程中, 都会发生Be的峰位漂移现象. 如张 文兰等人 ${ }^{[14]}$ 利用JXA-8800(JEOL)配置的LDEB晶体测 试绿柱石时, 金属Be的峰位 $L=221.7 \mathrm{~mm}$, 而绿柱石中 $\mathrm{Be}$ 的峰位 $L=229.1 \mathrm{~mm}$, 向右漂移了 $7.4 \mathrm{~mm}$. 当束流增 加到 $150 \mathrm{nA}$ 时, 其Be的峰位 $L=231.9 \mathrm{~mm}$, 漂移了 $10.2 \mathrm{~mm}$ (表2), 可见漂移的程度与束流大小有关. 本研 究实验所用的JXA-8100(JEOL)上配置的LDE3H晶体

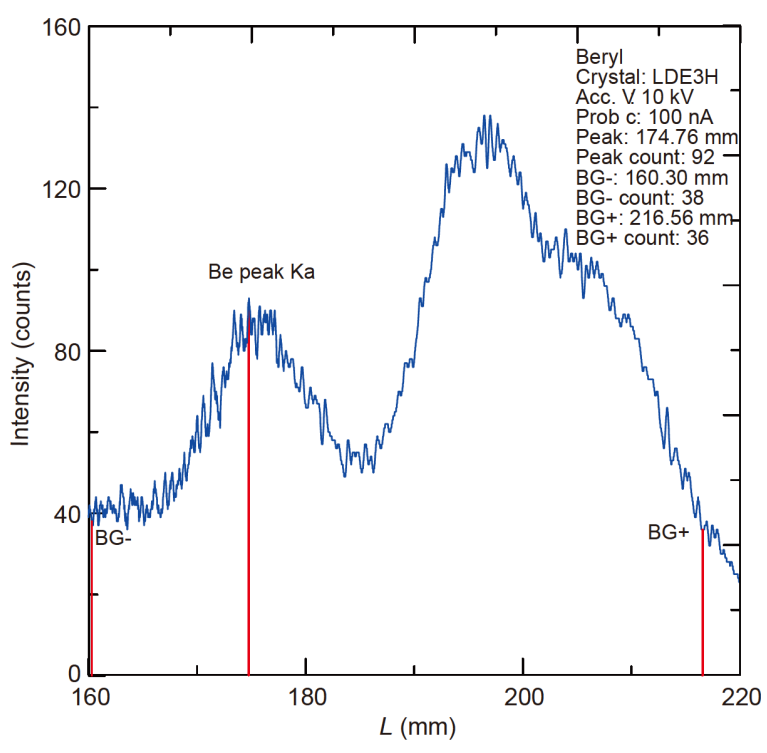

图 3 (网络版彩色)绿柱石波谱扫描图谱及峰位和上下背景(LDE3H 晶体)

Figure 3 (Color online) The peak position, upper background position, and lower background position of Be for the beryl sample by spectral scanning with LDE3H crystal

在通过元素波谱扫描后获得金属 $\mathrm{B}$ 的峰位 $L=$ $168.80 \mathrm{~mm}$, 绿柱石中Be的峰位则为 $L=174.76 \mathrm{~mm}$, 漂移 了 $5.96 \mathrm{~mm}$. 浙江大学吴润秋和饶灿 ${ }^{[6]}$ 展示了他们对含 $\mathrm{Be}$ 矿物最新的EPMA分析成果. 所用仪器为岛津 EPMA-1720H, 分光晶体为LSA300. 其中对金属Be和5 种含 $\mathrm{Be}$ 矿物进行 $\mathrm{Be}$ 寻峰发现, 矿物中Be的峰位都有不 同程度的向右漂移现象, 这种现象也称为谱线的化学 漂移 ${ }^{[16]}$.

峰位漂移是向着低能端的方向漂移, 其机理为元 素 $\mathrm{K} \alpha$ 特征 $\mathrm{X}$ 射线辐射是由 $\mathrm{K}-\mathrm{L}$ 两层之间的电子跃迁产 生. 对于原子序数小于 10 的超轻元素而言, 核外电子只 有 $\mathrm{K}$ 和 $\mathrm{L}$ 两层. 在形成化合物时, L层电子要参与反应, 因此它在形成不同的化合物时，L层电子的能量也不 同，从而使其特征X射线谱型因化合物不同而异 ${ }^{[16]}$. 而 对于 4 号元素Be而言, 核外电子只有 $\mathrm{K}$ 层, 且在 $1 \mathrm{~S}$ 和 $2 \mathrm{~S}$ 轨道上各有 2 个电子, 当高压电子束对 $\mathrm{Be}$ 元素激发时, $2 \mathrm{~S}$ 轨道上的电子向 $2 \mathrm{P}$ 轨道发生跃迁后处于激发状态, 如果没有其他元素参与, 这种激发态电子将回迁到原 始的 $2 \mathrm{~S}$ 轨道，回迁过程将以X-射线方式将跃迁时吸收 的能量释放出来, 其Be的峰位不会发生漂移. 在含 $\mathrm{Be}$ 矿物中, 当 $\mathrm{Be}$ 遇到 $\mathrm{O}$ 元素, 而是以共价键的方式形成 $\mathrm{BeO}$ 化合物，在此过程中原来处于激发态 $2 \mathrm{P}$ 轨道上的 2 个电子, 不能再回到原始的 $2 \mathrm{~S}$ 轨道, 而是以 Be-O原子 

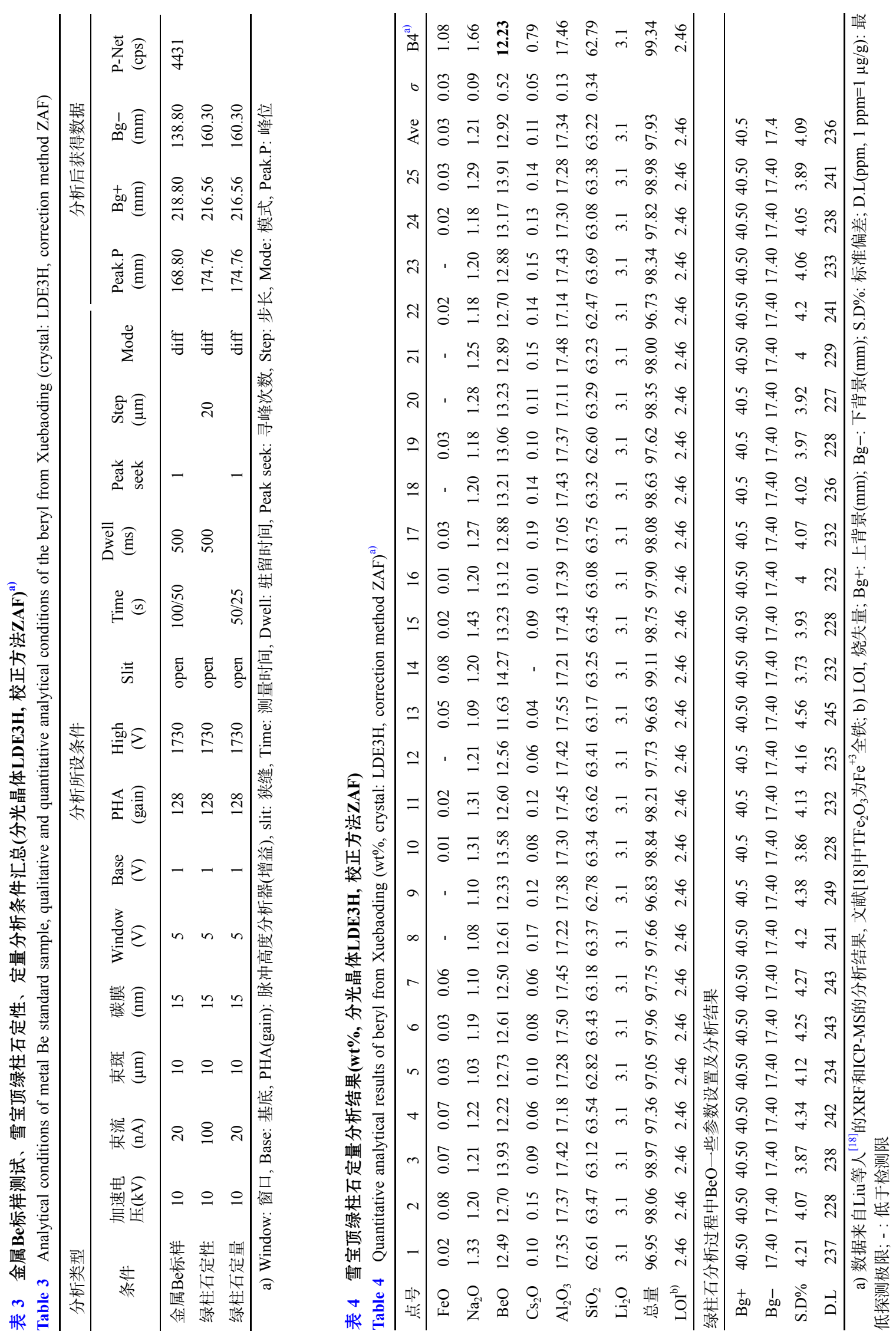
轨道杂化的方式形成一种新的分子轨道, 这种轨道其 所处能量级介于 $2 \mathrm{~S}$ 和 $2 \mathrm{P}$ 之间, 由于其能量低于激发态 (2p) 而使得Be-O也更加趋于稳定 ${ }^{[20]}$. 原子轨道杂化是 导致Be元素峰位向低能端漂移形成机理, 在全元素波 谱扫描图上, 如果横坐标为 $L$ 或 $\lambda$ 时, 峰位向右漂移, 如 果横坐标为 $E_{\mathrm{eV}}$ 时, 则峰位向左侧低能端漂移 $(L$ 与 $\lambda$ 二者 为正相关关系, $\lambda$ 与 $E$ 为负相关关系). 原子轨道杂化的 结果是形成新的分子轨道, 由此很好地解释了为什么 峰位向着低能量方向漂移. 至于峰位漂移的程度, 则取 决于Be矿物中所含元素的种类、矿物的晶体结构, 以 及测试过程中束流的大小等因素.

\section{2 数据质量讨论}

表4是利用JXA-8100、分光晶体LDE3H对雪宝顶 绿柱石 25 个点连续测量结果. 从表中数据可知, $\mathrm{BeO}$ 的 含量在 $11.63 \mathrm{wt} \% \sim 14.27 \mathrm{wt} \%$ 之间, 25 个数据中, 只有 1 个 数据大于 $14 \mathrm{wt} \%$ (14号点)和 1 个小于 $12 \mathrm{wt} \%$ (13号点), 其 他 23 个点 $\mathrm{BeO}$ 含量在 $12.22 \mathrm{wt} \% \sim 13.93 \mathrm{wt} \%$ 之间, 占总量 的 $92 \%$, 其他元素都比较稳定, 表明仪器状态很稳定.

表4中给出了每个点的Be X-射线强度计数的标准 偏差S.D $(\%)$ (standard deviation: Counting error \% of Xray intensities)和最低探测极限(D.L ppm). 根据下面 $S . D$ (\%)计算公式 ${ }^{[21]}$ :

$S . D(\%)=\frac{100}{I_{\text {net }}} \sqrt{\frac{I_{\text {peak }}}{t_{\text {peak }}}+\left[\overline{\frac{L_{\mathrm{PBH}}}{L}}\right]^{2} \frac{I_{\mathrm{PBL}}}{t_{\mathrm{PBL}}}+\left[\frac{L_{\mathrm{PBL}}}{L}\right]^{2} \frac{I_{\mathrm{PBH}}}{t_{\mathrm{PBH}}}}$,

增加峰位、上下背景计数时间 $\left(t_{\text {peak }} 、 t_{\mathrm{PBH}} 、 t_{\mathrm{PBL}}\right)$ 和元素 的净计数 $I_{\mathrm{net}}$, 可以降低 $S . D(\%)$ 值, 提高数据的精度.

表4中给出 25 个点中BeO的 $S . D(\%)$ 在 $3.73 \% \sim 4.56 \%$ 之间, 平均为 $4.09 \%$. 对于超轻元素 Be 而言, 由于其计 数率很低, 加之强烈的吸收效应不能设置太长的测量 时间, 获得这样的 S.D (\%)数据是不错结果. 表 4 中 25 个 点 $\mathrm{BeO}$ 的 $\sigma=0.52$, 在对超轻元素Be测试而言, 也应属于 不错的结果. $\sigma$ 值受分析数据的量 $(n)$ 影响, 增加测试的 数量会降低 $\sigma$ 值.

元素的最低检测限 $(D . L)$ 由统计的计算公式给出, 其数值与单点分析偏差接近. 检测限主要与峰背比有 关, 电压、电流、峰位计数时间、峰位及背景选择的 准确性都会影响检测限的数值. 在其他元素相同的情 况下, 增加峰位测试时间可以降低检测限.

表 4 中给出了 25 个点Be的最低探测极限值 $(1 \sigma$ 时), 在225 246 ppm之间, 平均为 $232 \mathrm{ppm}$ (测试时间峰位
$50 \mathrm{~s}$, 背景 $25 \mathrm{~s})$. 与表 2 中相同电流 $(20 \mathrm{nA})$ 、相同束斑 直径 $(10 \mu \mathrm{m})$ 的 D. $L$ 值( 3 个点 $10070,9863,7527$, 平均 $9153 \mathrm{ppm}$, 测试时间峰位 $100 \mathrm{~s}$, 背景 $50 \mathrm{~s}$ ) 相比较, 232 ppm远远好于 9153 ppm, 进一步说明了利用LDE3H 测Be比LDEB有着巨大的优势.

\section{3 强烈的吸收效应}

原子序数越高的元素, 质量吸收效应越低, 相反原 子序数越低的元素其质量吸收效应越高. 例如: Fe的 $\mathrm{K} \alpha$ 质量吸收系数为 100 , 最大 700 , 而 $\mathrm{N}$ 的 $\mathrm{K} \alpha$ 质量吸收 系数为 10000 , 最大为 35000 . 对于比 $N$ 更轻的元素Be而 言, 其质量吸收效应更加显著, 最高可达 $125300^{[22]}$. 在 EPMA分析过程中, Be矿物中所含有的其他元素都比 $\mathrm{Be}$ 原子序数大, 因此除了 $\mathrm{Be}$ 本身计数率很低外, 还有 一大部分的计数被其他元素所吸收, 这也是导致含 $\mathrm{Be}$ 矿物很难获得准确数据最主要的因素. 对于超轻元素 而言, 加速电压和束流的变化都会影响吸收效应. 也 就是在定量分析过程中, 使用 ZAF 修正法时, 其中对 吸收效应 A 的修正是获取理想数据的保证. 图4(a), (b) 更加形象地表明吸收效应对含 Be矿物分析影响最大 的因素.

从图4可以看出, 不论是在X-射线产生的深度、强 度以及 X射线产生的最强深度, 金属 $\mathrm{Be}$ 和 $\mathrm{BeO}$ 都有较大 的区别. 图4(a)为金属Be在 $10 \mathrm{kV}, 100 \mathrm{nA}$ 条件下 $\mathrm{Be} \mathrm{K} \alpha$ 特征X射线产生深度约为 $1500 \mathrm{~nm}$ (generated蓝色曲线), 同时辐射到样品表面的深度与其产生的深度几乎相等, 也在 $1500 \mathrm{~nm}$, 这意味着金属Be由于没有其他元素吸 收, 所产生的特征X射线几乎能够全部辐射到样品表面 而被X-射线探头收集(红色曲线). X射线产生最集中的 深度约为 $980 \mathrm{~nm}$.

图4(b)是在相同条件下, $\mathrm{BeO}$ 中 Be的特征X射线产 生与辐射到样品表面轨迹模拟结果, 其产生的深度明 显大于金属 $\mathrm{Be}$ ，约为 $1800 \mathrm{~nm}$ (generated蓝色曲线). 同 时辐射到样品表面的深度约在 $550 \mathrm{~nm}$, 远远小于产生 的深度, 约占总深度的 $30 \%$. 这意味着 $\mathrm{BeO}$ 中产生的 $\mathrm{Be}$ 的 $\mathrm{K} \alpha$ 射线只有约 $30 \%$ 辐射后被X-射线探头收集(红色 曲线), 而其余约70\%则被 $\mathrm{O}$ 元素所吸收, $\mathrm{X}$ 射线产生最 集中的深度约为 $1040 \mathrm{~nm}$.

至于 $\mathrm{Be}$ 的特征X-射线被吸收的程度, 则取决于含 Be矿物中其他元素的种类, 如 $\mathrm{Li}, \mathrm{O}, \mathrm{Na}, \mathrm{Mg}, \mathrm{Si}, \mathrm{Al}$ 这6 种元素对Be的质量吸收效应最大 ${ }^{[22]}$, 故除了 $\mathrm{Mg}$ 元素之 外, 其他 5 种元素都是本次研究样品绿柱石中的常量元 

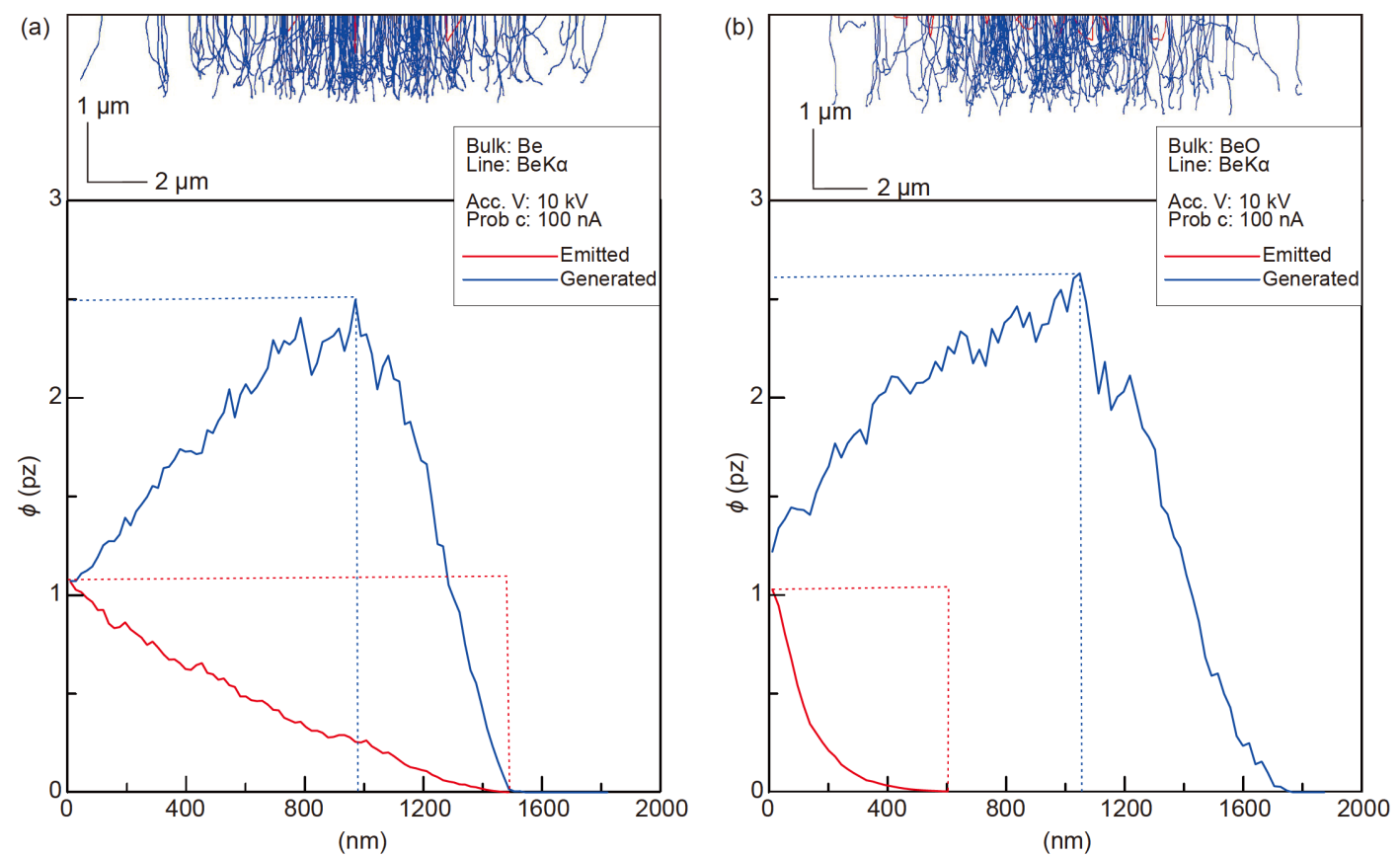

图 4 (网络版彩色) $10 \mathrm{kV}, 100 \mathrm{nA}$, 金属 $\mathrm{Be}$ 和BeO中Be Ka线Monte Carlo模拟 ${ }^{[23]}$. (a) 金属Be的Be Ka线Monte Carlo模拟轨迹线及随深度变化的 PRZ曲线; (b) BeO中 Be Ka线Monte Carlo模拟轨迹线及随深度变化的PRZ曲线. 模拟软件选择WinXRay 1.4.2, 软件介绍见文献[23]

Figure 4 (Color online) Monte Carlo simulation displaying of the characteristic $\mathrm{X}$-ray for $\mathrm{Be} \mathrm{K} \alpha$ of $\mathrm{Be}$ and $\mathrm{BeO}$ at $10 \mathrm{kV}$ accelerating voltage on 20 nA beam current. (a) Monte Carlo simulation results of the characteristic X-ray trajectory and PRZ curve for Be Ka of metal Be. (b) Monte Carlo simulation results of the characteristic X-ray trajectory and PRZ curve for Be $\mathrm{K} \alpha$ of BeO. Simulation was performed using WinXRay 1.4.2 program ${ }^{[23]}$

素, 这也是为什么绿柱石难测的主要原因.

\section{4 对超轻元素定量测试加速电压与束流选择之 考虑}

Dyar等人 ${ }^{[13]}$ 使用CamecaSX-50(PC3), 加速电压 $10 \sim 15 \mathrm{kV}$, 相应束流为 $40 \sim 20 \mathrm{nA}$, 计数时间 $20 \sim 60 \mathrm{~s}$, 束 斑直径为 $5 \sim 10 \mu \mathrm{m}$ ，校正方法为PAP，对硅铍石 $(n=110$, $\sigma=0.77 ; n=40, \sigma=1.29)$ 和嗍铍石 $(n=26, \sigma=1.22)$ 两种矿物 进行了测试, 获得了比较理想结果.

Cheng等人 ${ }^{[24]}$ 曾经使用CamecaSX-100电子探针 (PC3 分光晶体), 在 $100 \mathrm{nA}$ 束流, 束斑 $5 \mu \mathrm{m}, 5$ 、 10、 $15 \mathrm{kV}$ 三种不同的电压下对一个电气石和 7 个含 $\mathrm{B}$ 硅酸盐玻璃进行了测试. 以 $5 \mathrm{kV}$ 加速电压、100 nA束 流, 束斑 $20 \mu \mathrm{m}$ 的测试条件, 对 $\mathrm{B}$ 进行单独测试. 并使用 了PHA对干扰峰进行了过滤, 其中 $5 \mathrm{kV}$ 获得的结果最 佳，测出了 $\mathrm{B}_{2} \mathrm{O}_{3}$ 含量仅为 $0.18 \mathrm{wt} \%$ 、其 $\sigma$ 值为 0.08 最佳 结果, 将超轻元素B的测试提高到了一个很高的水平.

吴润秋和饶灿 ${ }^{[6]}$ 使用岛津 (Shimadzu) 电子探针 (LSA300分光晶体). 其加速电压为 $12 \mathrm{kV}$, 无水铍矿物 束流为 100 200 nA, 对含水铍矿物束流为50 100 nA, 选择 PHA过滤干扰线系, 使用福建南平的硅铍石和四
川平武绿柱石矿物做标样. 从所给出的 $\mathrm{BeO}$ 数据看, 也 取得了满意的结果 ${ }^{[6]}$. 但是由于数据点较少, 这些点是 否为连续测试获得，以及缺少 $\sigma$ 值，对测试条件还有进 一步优化的可能.

本研究使用JXA-8100(JEOL)电子探针(LDE3H分 光晶体), $10 \mathrm{kV}$ 加速电压, $20 \mathrm{nA}$ 束流, 通过修改PHA中 的Base值和window值, 选择合适的上下背景值, 使用金 属 Be标样, 对绿柱石定量分析也获得了满意的结果.

从上述几个例子看，对超轻元素Be或 $\mathrm{B}$ 的测试条 件, 加速电压和束流的设置, 并没有唯一标准. 一般情 况下低电压与大束流、高电压与小束流配合，再对其 条件进行合适的设置, 就能获得好的测试结果, 或是将 超轻元素和普通元素分开测量. 总之在能获得满意的 测试结果时, 尽量选择低束流, 或低电压, 以此尽量减 少其他元素对Be的吸收效应.

\section{7 结论}

通过对可能影响电子探针绿柱石中Be元素测试结 果诸因素的分析与讨论表明: (1) 使用JEOL 电子探针 配备的LDE3H分光晶体的分析结果，明显好于用配备 $\mathrm{LDEB}$ 分光晶体, 其原因不论是晶体的 $2 d$ 值 
(LDE3H: $2 d=20 \mathrm{~nm}$, LDEB: $2 d=14.5 \mathrm{~nm}$ ), 还是Be的 $L$ 值, LDE3H的 $L=168.80 \mathrm{~mm}$ 靠近罗兰圆中角度，计数强度 较好的位置, 利于上下背景的选取; LDEB的 $L=221.7$ $\mathrm{mm}$, 峰位的 $L$ 值大, 属于高布拉格角位置, 该位置不但 计数强度低, 而且不利于上背景的选取. (2) 加速电压 设置为 $10 \mathrm{kV}$, 束流为 $20 \mathrm{nA}$ 的测试条件, 既能满足Be元 素的测试, 同时也能满足绿柱石中其他元素的测试. 如 果遇到Be含量更低的铍矿物, 应使用低电压将Be元素 单独测试, 其他元素用普通条件测试, 效果会更好. (3)
把PHA gain(增益)设置为 $128, \operatorname{slit}$ (狭缝)设置为open, 都 是为了尽量多收集Be的特征 $\mathrm{X}$ 射线, 并把脉冲的信号放 大, 为获得理想的测试结果提供保证. (4) Base设置为 1 , window设置为 5 及微分模式(differential mode)也是获得 好的测试结果的必要条件.

总之，从目前已发表的关于超轻元素Be的定量分 析来看, 不同用户所用仪器不同, 尤其是所配分光晶体 和修正方法不同, 导致目前很难将EPMA测试Be的条件 统一, 只能是具体情况具体分析, 才能获得理想的结果.

致谢两位审稿专家对本文提出了很多非常中肯和建设性的意见和建议; 中国地质科学院地质研究所刘琰博士提供了宝贵的 绿柱石样品, 在实验过程中与台湾清华大学蔡淑月老师进行了有意义讨论, 在此一并感谢.

\section{参考文献}

1 Department of Earth Science, Nanjing University. Geochemistry (in Chinese). Nanjing: Nanjing University Press, 1979.514 [ 南京大学地质系. 地 球化学. 南京: 南京大学出版社, 1979. 514]

2 Taylor S R, McLennan S M. The geochemical evolution of the continental crust. Rev Geophys, 1995, 33: 241-265

3 Asian Metal. Beryllium property (in Chinese). http://baike.asianmetal.cn/metal/be/characteristic.shtml [亚洲金属网. 铍的性质. http://baike. asianmetal.cn/metal/be/characteristic.shtml]

4 Kupriyanova I I, Rumyantseva G Y. Forms of occurrence of beryllium in technogenic halos around the Yaroslavl Mining and benefication plant (Maritime Region). Geochim Int, 1994, 2: 73-86

5 Liang F, Zhao T, Wang D H, et al. Supply and demand forecast ang development strategy beryllium resources in China (in Chinese). China Min, 2018, 11: 6-7 [梁飞, 赵汀, 王登红, 等. 中国铍资源供需预测与发展战略. 中国矿业, 2018, 11: 6-7]

6 Wu R Q, Rao C. EPMA analysis of ultralight metal Be (in Chinese). Chin Sci Bull, 2020, 65: 2161-2168 [吴润秋, 饶灿. 超轻元素金属铍的电子 探针分析. 科学通报, 2020, 65: doi: 2161-2168]

7 Guo Y J, Wang R C, Xu S J, et al. A study of structure of a rare tabular crystal of beryl (in Chinese). Geol Rev, 2000, 3: 312-317 [郭延军, 王汝成, 徐士进, 等. 板柱状绿柱石晶体的结构测定. 地质论评, 2000, 3: 312-317]

8 Guo Y J, Wang R C, Xu S J. Vibrational spectra of beryl from Xubaoding, Pingwu County, Sichuan Province (in Chinese). Geol J China Univ, 2000, 2: 201-204 [郭延军, 王汝成, 徐士进. 四川平武雪宝顶绿柱石的振动光谱. 高校地质学报, 2000, 2: 201-204]

9 Wang R C, Che X D, Zhang W L, et al. Geochemical evolution and late re-equilibration of NaCs-rich beryl from the Koktokay \#3 pegmatite (Altai, NW China). Eur J Miner, 2009, 21: 795-809

10 Che X D, Wang R C, Hu H, et al. Beryllium mineralization in the Yichun topaz-lepidolite granite, Jiangxi: Associations of beryllium phosphate minerals (in Chinese). Acta Petrol Sin, 2007, 6: 1552-1560 [车旭东, 王汝成, 胡欢, 等. 江西宜春黄玉-锂云母花岗岩中的铍矿化作用铍磷酸盐 矿物组合. 岩石学报, 2007, 6: 1552-1560]

11 Uher P, Chudík P, Bačík P, et al. Beryl composition and evolution trends: An example from granitic pegmatites of the beryl-columbite subtype, Western Carpathians, Slovakia. J Geosci, 2010, 55: 69-80

12 Wang R C, Wu F Y, Xie L, et al. A preliminary study of rare-metal mineralization in the Himalayan leucogranite belts, South Tibet. Sci China Earth Sci, 2017, 60: 1655-1663 [王汝成, 吴福元, 谢否, 等. 藏南喜马拉雅淡色花岗岩稀有金属成矿作用初步研究. 中国科学: 地球科学, 2017, 47: $871-880]$

13 Dyar M D, Wiedenbeck M, Robertson D, et al. Reference minerals for the microanalysis of light elements. Geostand Geoanal Res, 2001, 25: 441463

14 Zhang W L, Wang R C, Cai S Y. EPMA quantitative analysis of super-light element Be: As a sample of beryl (in Chinese). J Chin Electr Microsc Soc, 2006, 25(Suppl): 293-294 [张文兰，王汝成，蔡淑月. 超轻元素Be元素电子探针定量分析一一以绿柱石为例. 电子显微学报，2006, 25(Suppl): 293-294]

15 Zhao T X, Chen W D, Yin T, et al. EPMA quantitative analysis of beryllium mineral beryl (in Chinese). Acta Miner Sin, 2019, 39: 1-7 [赵同新, 陈 文迪, 殷婷, 等. 电子探针对含Be矿物绿柱石的定量分析. 矿物学报, 2019, 39: 1-7]

16 Xu L Y, Shang Y H, Guo Y F, et al. Some aspects for quantitative analysis of ultralight element in EPMA (in Chinese). J Instrum Anal, 1994, 5: 37- 
41 [徐乐英, 尚玉华, 郭延风, 等. 用电子探针定量分析超轻元素中的几个问题. 分析测试学报, 1994, 25: 37-41]

17 Zhou J X, Mao S H. Electron Probe Analysis (in Chinese). Beijing: Geological Publishing House, 1988. 388 [周剑雄, 毛水和. 电子探针分析. 北 京: 地质出版社, 1988. 388]

18 Liu Y, Deng J, Shi G H, et al. Geochemical and morphological characteristics of coarse-grained tabular beryl from the Xuebaoding W-Sn-Be deposit, Sichuan Province, western China. Int Geol Rev, 2012, 54: 1673-1684

19 Zhang R X, Yang S Y. A mathematical model for determining carbon coating thickness and its application in electron probe microanalysis. Microsc Microanal, 2016, 22: 1374-1380

20 Fu X C. University Chemistry (in Chinese). Beijing: Higher Education Press, 1999. 546-573 [傅献彩. 大学化学. 北京: 高等教育出版社, 1999. 546-573]

21 Japan Electronics Co., Ltd. Serving Advanced Technology. Quantitative Analysis Software. 2005/10, 2-93

22 Japan Electronics Co., Ltd. Quantitative Analysis with Electron Probe Micro-analyzar Apendixes, 1985. 60

23 Demers H, Horny P, Gauvin R, et al. WinX-ray: A new Monte Carlo program for the simulation of X-ray and charging materials. Microsc Microanal, 2002, 8: 1498-1499

24 Cheng L, Zhang C, Li X, et al. Improvement of electron probe microanalysis of boron concentration in silicate glasses. Microsc Microanal, 2019, 25: $874-882$ 


\title{
Optimum conditions for quantitative analysis of beryllium by electron probe microanalysis: A case study of beryl
}

\author{
Wenlan Zhang ${ }^{1 *}$, Xudong Che ${ }^{1}$, Rucheng Wang ${ }^{1}$, Lei Xie ${ }^{1}$, Xiaofeng Li $^{2,3,4}$ \& Di Zhang \\ ${ }^{1}$ State Key Laboratory for Mineral Deposits Research, School of Earth Sciences and Engineering, Nanjing University, Nanjing 210023, China; \\ ${ }^{2}$ Laboratory of Mineral Resources, Institute of Geology and Geophysics, Chinese Academy of Sciences, Beijing 100029, China; \\ 3 Innovation Academy for Earth Science, Chinese Academy of Sciences, Beijing 100029, China; \\ ${ }^{4}$ College of Earth and Planetary Sciences, University of Chinese Academy of Sciences, Beijing 100049, China; \\ ${ }^{5}$ State Key Laboratory of Lithospheric Evolution, Institute of Geology and Geophysics, Chinese Academy of Sciences, Beijing 100029, China \\ * Corresponding author, E-mail: zhangwl@nju.edu.cn
}

Beryllium $(\mathrm{Be})$ is resistant to high temperatures and corrosion, and thus is an important strategic resource for use in defense and military industries. Beryllium is also used in nuclear reactors as a neutron reflector or moderator. At present, there are numerous unresolved research questions regarding Be resources, including the mechanisms of Be mineralization, $\mathrm{Be}$ occurrence in minerals, and Be separation and extraction from ores. Therefore, it is important to be able to measure $\mathrm{Be}$ contents of minerals in situ by electron probe microanalysis (EPMA). However, given that Be is an ultra-light element, it is the most difficult element to measure by EPMA. The objectives of this research were to: (1) develop robust techniques for EPMA of Be-bearing minerals to support research into Be resources; and (2) advance the techniques for the measurement of ultra-light elements.

In this study, beryl samples from the Xuebaoding deposit in Sichuan Province, China, were selected for Be analysis by EPMA. Data from two different electron microprobes (JEOL JXA-8800 and JXA-8100) with different crystals (LDEB and LDE3H, respectively) were compared. Our results show that the LDE3H crystal is better for Be analysis than the LDEB crystal, as the interplanar spacing of the former $(2 d=20 \mathrm{~nm})$ is much larger than that of the latter $(2 \mathrm{~d}=14 \mathrm{~nm})$. Under appropriate measurement conditions, satisfactory Be data can be obtained with the LDE3H crystal. The highest peak count for the Be Ka line in the Be metal sample was obtained at an accelerating voltage of $10 \mathrm{kV}$ (higher than at 3, 5, or $15 \mathrm{kV}$ ). The highest peak-to-background ratio for the Be Ka line in the beryl sample was also obtained at an accelerating voltage of $10 \mathrm{kV}$, a probe current of $100 \mathrm{nA}$, and beam size of $10 \mu \mathrm{m}$. The highest peak-to-background ratio for the $\mathrm{Be} \mathrm{Ka}$ line in the beryl sample was obtained at a probe current of $20 \mathrm{nA}$ (higher than at 10,50,100, or $200 \mathrm{nA}$ ) with an accelerating voltage of $10 \mathrm{kV}$ and beam size of $10 \mu \mathrm{m}$. Thus, the optimum analytical conditions are as follows: accelerating voltage $=10 \mathrm{kV}$; beam current $=20 \mathrm{nA}$; beam size $=0-10 \mu \mathrm{m}$; PHA (gain) $=128$; base $=1$; window $=5$; slit open; differential mode; standard=Be metal; thickness of carbon film for Be metal standard and beryl sample $=15 \mathrm{~nm}$; background=avoidance of interference peaks of other elements close to the Be peak position in high order; ZAF correction.

Based on the chemical characteristics of Be, we also investigated why Be is challenging to measure by EPMA, and considered the chemical peak shift during analysis and the selection and optimization of the analytical conditions. The peak shifts for different Be minerals are caused mainly by the hybridization of atomic orbits between $\mathrm{Be}$ and $\mathrm{O}$ atoms. The absorption effect is the main reason why Be analyses are challenging by EPMA. The absorption effect is mainly due to $\mathrm{O}$ atoms, as well as other elements in minerals, such as $\mathrm{Si}, \mathrm{Na}, \mathrm{Mg}$, and $\mathrm{Li}$. A uniformly and accurately controlled carbon film thickness for the standard and samples is a key factor. A digital coating instrument (LEICA-EM-ACE200) was used in this work to ensure a precise finish. A compilation of published Be data obtained with JEOL, Shimadzu, and Cameca EMPA instruments shows that each has different crystals (LDE3H, LSA300, and PC3, respectively) and conditions for optimal Be analysis. In addition, the optimal peak position, accelerating voltage, and beam current vary for different Be Bearing minerals analyzed with the same EPMA instrument. However, a low beam current and accelerating voltage generally minimize the absorption effect on Be.

electron probe microanalysis (EPMA), quantitative analysis, ultralight beryllium element, beryl, analytical condition, absorption effect

doi: 10.1360/TB-2020-0316 